\title{
Synthesis Charcterization and Antimicrobiological Activity of 4-Thiazolidinone Derivatives with Furan and Pyridine Moeities
}

\author{
Hiteshkumar J. Gujjar ${ }^{1}$, Dr.Sheetal Gulati ${ }^{2}$ and Dr.H.S.Patel ${ }^{3}$ \\ Research Scholar, Rabindranath Tagore University, Bhopal (M.P.) India ${ }^{1}$ \\ Professor, Department of Chemistry, Rabindranath Tagore University, Bhopal (M.P.) India ${ }^{2}$ \\ Ex.Head and professor, Department of Chemistry, Sardar Patel University, Vallabh Vidyanagar (Gujarat) India ${ }^{3}$
}

\begin{abstract}
Isoniazid (1) on reaction with 5-arylfuran-2-carboxaldehydes (2a-e) yield N'-((5-Arylfuran-2-yl) methylene) isonicotinohydrazide (3a-e). Post reaction of these hydrazones (3a-e) with mercapto acetic acid afforded N-(4-oxo-2-(5Arylfuran-2-yl) thiazolidin-3-yl) isonicotinamide (4a-e). Such 4-thiazolidinone derivatives were then treated with phenyl sulphonamide diazonium chloride yielded the compounds (N-(2-(5-arylfuran-2-yl)-4-oxo-5-(2-(4sulfamoylphenyl) hydrazono) thiazolidin-3-yl) isonicotinamide (5a-e). The structures of these series of heterocycles were assigned by analytical and spectral feature. All the were also evaluated for their antibacterial and antifungal activities.
\end{abstract}

Keywords: Isoniazid, Schiff base, thiazolidine, diazonium salt, spectroscopy antibacterial and antifungal activities.

\section{INTRODUCTION}

An azomethine group (-CH=N-) (known as Schiff base) is an intermediate for heterocycles compounds with good pharmacological activity etc ${ }^{1-6}$. One of the compound, furan-2-carbaldehydes in an agriculture renewal product possess a carbonyl group as a reactive centre. There are numbers of products can form via this intermediate ${ }^{7-10}$. The with known anti T. B. drug say Isoniazid can form Schiff base with furan aldehyde. The 4-thiazolidinones derivatives based on Schiff bases has not repeated so far. However, 4-Thiazolidinone and its derivatives exhibit various pharmacological properties $^{11-16}$. Hence, it was thought to merge 4- thiazolidinone with isoniazid and furan moieties. This may which may enhance the drug activity up to some extent, some of the above mentioned biological activities. Thus the present communication comprises the study of new derivatives of Isoniazid - thiazolidinone - furan system.. The whole synthetic approach schematically drown as follow (Scheme-I) 
Vol. 6, Issue 1, January 2019

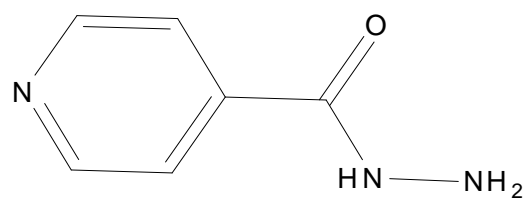

Isoniazide(1)

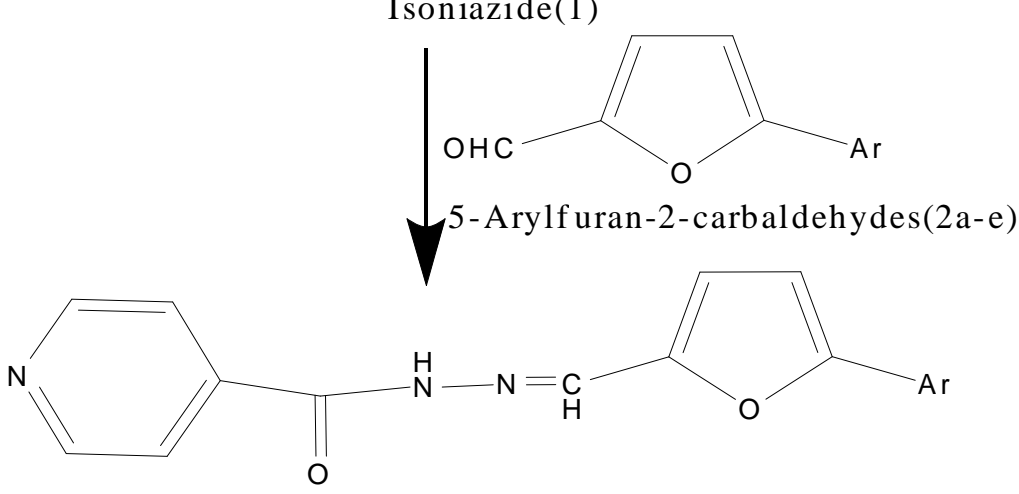

$N^{\prime}-((5-A r y l f$ uran-2-yl)methylene)isonic otinohydrazide( 3 a-e)

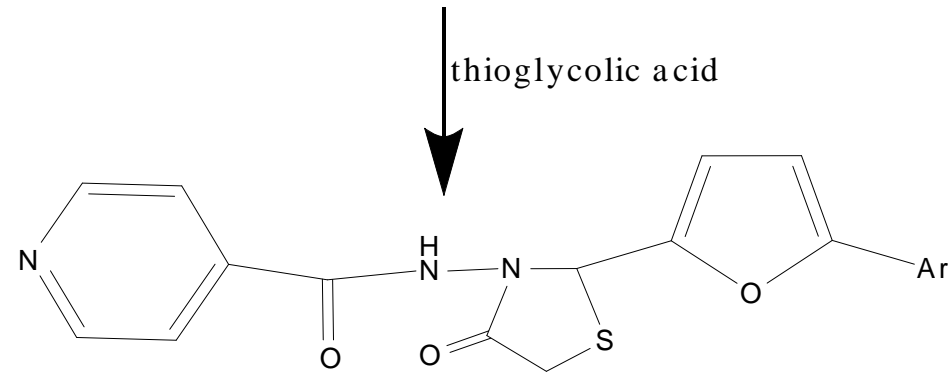

$N$-(4-oxo-2-(5-A rylf uran-2-yl)thiazolidin-3-yl)isonicotinamide (4a-e)

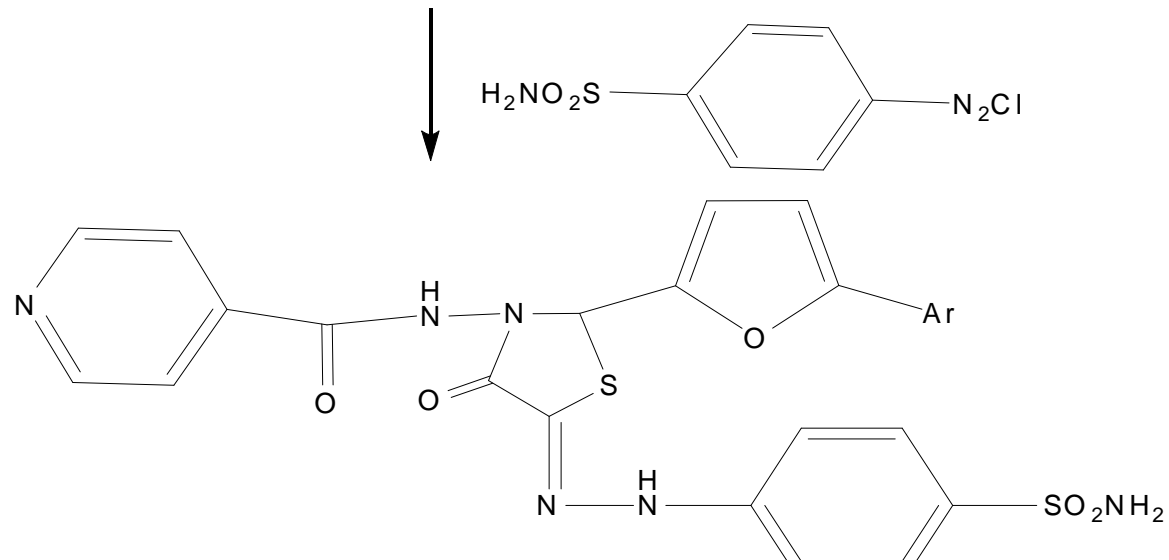

$(N-(2-(5-a r y l f$ ura n-2-yl)-4-ox o-5-(2-(4-sulf am oylphenyl)hydrazono $)$ thiazolidin-3-yl)isonicotinam ide (5a-e)
Where, $\mathrm{Ar}=$
(a) $\mathrm{C}_{6} \mathrm{H}_{5}$
(b) $4-\mathrm{CH}_{3}-\mathrm{C}_{6} \mathrm{H}_{4}$
(c) $4-\mathrm{Cl}-\mathrm{C}_{6} \mathrm{H}_{4}$
(d) $4-\mathrm{NO}_{2}-\underset{\mathbf{S C H}}{\mathrm{CH}_{6} \mathrm{H}_{4}} \mathbf{E M} \mathbf{- 1}-\mathbf{1}$

\section{EXPERIMENTAL}

\section{Material and Methods}

The $\mathrm{C}, \mathrm{H}, \mathrm{N}$ - content of sample were determine by carlo erba $\mathrm{C} \mathrm{H} \mathrm{N}$ analyser. The IR spectra of all compound were recorded in $\mathrm{KBr}$ pellets on a Nicolet 400D spectrometer and $1 \mathrm{H}$ NMR spectra were recorded in deuterated DMSO on a 


\section{International Advanced Research Journal in Science, Engineering and Technology}

Vol. 6, Issue 1, January 2019

Bruker spectrometer at $400 \mathrm{MHz}$. LC-MS of all samples taken on LC-MSD-Trap-SL_01046. All the chemical used were of pure grade.

\section{Preparation N'-((5-Arylfuran-2-yl)methylene) isonicotinohydrazide (3a-e)}

A suspension of Isoniazid (1), (10 mmol) and the 5-Arylfuran-2-carbaldehydes (2a-e) (10mmol) in ethanol (15ml) was refluxed on a water bath for $2 \mathrm{hrs}$. The pastry mass obtained. The liquid was decanted and then dry ether was added to get the solid powder it was air dried, and recrystallized R spirit .The characterization data of these compounds are given in Table -1 .

Table:-1 Characterization Data of Compounds (3a-e)

\begin{tabular}{|c|c|c|c|c|c|c|c|c|c|c|}
\hline \multirow{3}{*}{ Compd. } & \multirow{3}{*}{$\begin{array}{c}\text { Molecular } \\
\text { formula } \\
\text { (Mol.wt.) }\end{array}$} & \multirow{3}{*}{$\begin{array}{c}\text { LC- } \\
\text { MS } \\
\text { Data }\end{array}$} & \multirow{3}{*}{$\begin{array}{l}\text { Yield } \\
\%\end{array}$} & \multirow{3}{*}{$\underset{{ }^{0} \mathrm{C}}{\text { M.P.* }}$} & \multicolumn{6}{|c|}{ Elemental Analysis } \\
\hline & & & & & \multicolumn{2}{|c|}{$\% \mathrm{C}$} & \multicolumn{2}{|c|}{$\% \mathrm{H}$} & \multicolumn{2}{|c|}{$\% \mathbf{N}$} \\
\hline & & & & & Found & Calcd. & Found & Calcd. & Found & Calcd. \\
\hline $3 a$ & $\begin{array}{c}\mathrm{C}_{17} \mathrm{H}_{13} \mathrm{~N}_{3} \mathrm{O}_{2} \\
(291)\end{array}$ & 294 & 88 & $\begin{array}{l}205- \\
207\end{array}$ & 70.0 & 70.09 & 4.4 & 4.50 & 14.4 & 14.42 \\
\hline $3 b$ & $\begin{array}{c}\mathrm{C}_{18} \mathrm{H}_{15} \mathrm{~N}_{3} \mathrm{O}_{2} \\
(305)\end{array}$ & 308 & 86 & $\begin{array}{l}210- \\
211\end{array}$ & 70.8 & 70.81 & 4.9 & 4.95 & 13.7 & 13.76 \\
\hline $3 c$ & $\begin{array}{c}\mathrm{C}_{18} \mathrm{H}_{12} \mathrm{~N}_{3} \mathrm{O}_{2} \mathrm{Cl} \\
(325.5)\end{array}$ & 337 & 79 & $\begin{array}{l}208- \\
209\end{array}$ & 62.6 & 62.68 & 3.6 & 3.71 & 12.8 & 12.90 \\
\hline $3 d$ & $\begin{array}{c}\mathrm{C}_{17} \mathrm{H}_{12} \mathrm{~N}_{4} \mathrm{O}_{4} \\
(336)\end{array}$ & 343 & 78 & $\begin{array}{l}214- \\
216 \\
\end{array}$ & 60.7 & 60.71 & 3.5 & 3.60 & 16.6 & 16.66 \\
\hline $3 e$ & $\begin{array}{c}\mathrm{C}_{18} \mathrm{H}_{12} \mathrm{~N}_{2} \mathrm{O}_{2} \mathrm{Cl}_{2} \\
(321)\end{array}$ & 336 & 80 & $\begin{array}{l}217- \\
219\end{array}$ & 60.1 & 60.19 & 3.3 & 3.37 & 7.7 & 7.80 \\
\hline
\end{tabular}

* Uncorrected

\section{Preparation of N-(4-oxo-2-(5-Arylfuran-2-yl)thiazolidin-3-yl) isonicotinamide(4a-e)}

A mixture derivatives (3a-e)(10mmol) and marcapto acetic acid (10 mmol) in Tetra hydro furan (THF) (30ml), Anhydrous $\mathrm{ZnCl}_{2}$ was added as pinch. The mixture was refluxed for $15 \mathrm{hrs}$. The solvent was then decanted to get a bulk product, which was dissolved in isopropanol and passed through a column of silica gel using isopropanol: THF $(8: 2 ; \mathrm{v} / \mathrm{v})$ mixture as eluent. The elute was concentrated and the product was isolated from as 4-thiazolidinones (4a-e). The characterization data of these compounds are given in Table -2 .

Table:-2 Characterization Data of Compounds (4a-e)

\begin{tabular}{|c|c|c|c|c|c|c|c|c|c|c|c|c|}
\hline \multirow{3}{*}{ Compd. } & \multirow{3}{*}{$\begin{array}{c}\text { Molecular } \\
\text { formula } \\
\text { (Mol.wt.) }\end{array}$} & \multirow{3}{*}{$\begin{array}{c}\text { LC- } \\
\text { MS } \\
\text { Data }\end{array}$} & \multirow{3}{*}{$\begin{array}{l}\text { Yield } \\
\%\end{array}$} & \multirow{3}{*}{${ }^{\mathbf{M}} \mathbf{C} .{ }^{*}$} & \multicolumn{6}{|c|}{ Elemental Analysis } & \multirow{2}{*}{\multicolumn{2}{|c|}{$\% \mathrm{~S}$}} \\
\hline & & & & & \multicolumn{2}{|c|}{$\% \mathrm{C}$} & \multicolumn{2}{|c|}{$\% \mathbf{H}$} & \multicolumn{2}{|c|}{$\% \mathrm{~N}$} & & \\
\hline & & & & & Found & Calcd. & Found & Calcd. & Found & Calcd. & Found & Calcd. \\
\hline $4 a$ & $\begin{array}{c}\mathrm{C}_{19} \mathrm{H}_{15} \mathrm{~N}_{3} \mathrm{O}_{3} \mathrm{~S} \\
(365)\end{array}$ & 372 & 69 & $\begin{array}{l}211- \\
212 \\
\end{array}$ & 62.4 & 62.45 & 4.1 & 4.14 & 11.4 & 11.50 & 8.7 & 8.78 \\
\hline $4 b$ & $\begin{array}{c}\mathrm{C}_{20} \mathrm{H}_{17} \mathrm{~N}_{3} \mathrm{O}_{3} \mathrm{~S} \\
(379)\end{array}$ & 388 & 60 & $\begin{array}{l}205- \\
207\end{array}$ & 63.3 & 63.31 & 4.5 & 4.52 & 11.0 & 11.07 & 8.4 & 8.45 \\
\hline $4 c$ & $\begin{array}{c}\mathrm{C}_{19} \mathrm{H}_{14} \mathrm{~N}_{3} \mathrm{O}_{3} \mathrm{SCl} \\
(399.5)\end{array}$ & 405 & 59 & $\begin{array}{c}166- \\
168\end{array}$ & 57.0 & 57.07 & 3.5 & 3.53 & 10.5 & 10.51 & 8.0 & 8.02 \\
\hline $4 d$ & $\begin{array}{c}\mathrm{C}_{19} \mathrm{H}_{14} \mathrm{~N}_{4} \mathrm{O}_{5} \mathrm{~S} \\
(410)\end{array}$ & 417 & 67 & $\begin{array}{l}149- \\
150 \\
\end{array}$ & 55.6 & 55.60 & 3.4 & 3.44 & 13.6 & 13.65 & 7.8 & 7.81 \\
\hline $4 \mathrm{e}$ & $\begin{array}{c}\mathrm{C}_{19} \mathrm{H}_{13} \mathrm{~N}_{3} \mathrm{O}_{3} \mathrm{~S}_{2} \mathrm{Cl} \\
(433)\end{array}$ & 447 & 64 & $\begin{array}{c}168- \\
169\end{array}$ & 52.5 & 52.55 & 3.0 & 3.02 & 9.6 & 9.68 & 7.3 & 7.38 \\
\hline
\end{tabular}

* Uncorrected

Preparation of (N-(2-(5-arylfuran-2-yl)-4-oxo-5-(2-(4-sulfamoylphenyl)hydrazono) thiazolidin-3-yl) isonicotinamide (5a-e)

The hydrazo derivatives were prepared by method reported for other 4-thiazolidine derivatives ${ }^{18}$

A solution of 4-thiazolidinone (4a-e) $(10 \mathrm{mmol})$ in isopropanol $(30 \mathrm{ml})$ and sodium acetate $(50 \mathrm{~g})$ was stirred in cold bath of temperature $0-5^{\circ} \mathrm{C}$. Cold diazotised solution $\left(0-5^{\circ} \mathrm{C}\right)$ of 4 -amino sulphonamide $(10 \mathrm{mmol})$ was added drop wise to this solution with good stirring $\left(0-5^{\circ} \mathrm{C}\right)$. The solid products were filtered off and air dried. M. P. $>250^{\circ} \mathrm{C}$, The characterization data projected in table 3. 
International Advanced Research Journal in Science, Engineering and Technology

Vol. 6, Issue 1, January 2019

Table:-3 characterization Data of Compounds (5a-e)

\begin{tabular}{|c|c|c|c|c|c|c|c|c|c|c|c|c|}
\hline \multirow{3}{*}{ Compd. } & \multirow{3}{*}{$\begin{array}{l}\text { Molecular } \\
\text { formula } \\
\text { (Mol.wt.) }\end{array}$} & \multirow{3}{*}{$\begin{array}{c}\text { LC- } \\
\text { MS } \\
\text { Data }\end{array}$} & \multirow{3}{*}{$\begin{array}{l}\text { Yield } \\
\%\end{array}$} & \multirow{3}{*}{$\underset{{ }^{0} \mathrm{C}}{\text { M.P.* }}$} & \multicolumn{6}{|c|}{ Elemental Analysis } & \multirow{2}{*}{\multicolumn{2}{|c|}{$\% \mathrm{~S}$}} \\
\hline & & & & & \multicolumn{2}{|c|}{$\% \mathrm{C}$} & \multicolumn{2}{|c|}{$\% \mathrm{H}$} & \multicolumn{2}{|c|}{$\% \mathrm{~N}$} & & \\
\hline & & & & & Found & Calcd. & Found & Calcd. & Found & Calcd. & Found & Calcd. \\
\hline $5 \mathrm{a}$ & $\begin{array}{c}\mathrm{C}_{25} \mathrm{H}_{20} \mathrm{~N}_{6} \mathrm{O}_{5} \mathrm{~S}_{2} \\
(548)\end{array}$ & 563 & 89 & $\begin{array}{l}261- \\
262\end{array}$ & 54.7 & 54.73 & 3.6 & 3.67 & 15.3 & 15.32 & 11.6 & 11.69 \\
\hline $5 b$ & $\begin{array}{c}\mathrm{C}_{26} \mathrm{H}_{22} \mathrm{~N}_{6} \mathrm{O}_{5} \mathrm{~S}_{2} \\
(562)\end{array}$ & 588 & 80 & $\begin{array}{l}255- \\
257\end{array}$ & 55.4 & 55.50 & 3.9 & 3.94 & 14.9 & 14.94 & 11.3 & 11.40 \\
\hline $5 \mathrm{c}$ & $\begin{array}{c}\mathrm{C}_{25} \mathrm{H}_{19} \mathrm{~N}_{6} \mathrm{O}_{5} \mathrm{~S}_{2} \mathrm{Cl} \\
(582.5)\end{array}$ & 596 & 89 & $\begin{array}{c}266- \\
268\end{array}$ & 51.4 & 51.50 & 3.2 & 3.28 & 14.3 & 14.41 & 10.9 & 11.00 \\
\hline $5 d$ & $\begin{array}{c}\mathrm{C}_{25} \mathrm{H}_{19} \mathrm{~N}_{7} \mathrm{O}_{7} \mathrm{~S}_{2} \\
(593)\end{array}$ & 512 & 87 & $\begin{array}{l}269- \\
270\end{array}$ & 50.5 & 50.58 & 3.2 & 3.23 & 16.5 & 16.52 & 10.7 & 10.80 \\
\hline $5 e$ & $\begin{array}{c}\mathrm{C}_{25} \mathrm{H}_{18} \mathrm{~N}_{6} \mathrm{O}_{5} \mathrm{~S}_{2} \mathrm{Cl}_{2} \\
(616)\end{array}$ & 631 & 84 & $\begin{array}{l}268- \\
269\end{array}$ & 48.6 & 48.63 & 2.9 & 2.94 & 13.5 & 13.61 & 10.3 & 10.39 \\
\hline
\end{tabular}

* Uncorrected

\section{BIOLOGICAL SCREENING}

Antimicrobial activities

The antimicrobial activities of all series of compounds (3a-e,4a-e,5a-e) were evaluated in terms of antibacterial and antifungal activities.

Table:-4 Antibacterial Activity of Compounds (3a-e)

\begin{tabular}{|c|c|c|c|c|}
\hline \multirow{2}{*}{ Compounds } & \multicolumn{2}{|c|}{ Gram +Ve } & \multicolumn{2}{c|}{ Gram - Ve } \\
\cline { 2 - 5 } & $\begin{array}{c}\text { Staphylococcus } \\
\text { aureus }\end{array}$ & $\begin{array}{c}\text { Bacillus } \\
\text { subtilis }\end{array}$ & E.coli & $\begin{array}{c}\text { Klebsiella } \\
\text { promioe }\end{array}$ \\
\hline 3a & 55 & 54 & 57 & 49 \\
\hline 3b & 56 & 53 & 52 & 57 \\
\hline 3c & 58 & 54 & 66 & 53 \\
\hline 3d & 62 & 59 & 58 & 52 \\
\hline 3e & 69 & 68 & 78 & 60 \\
\hline
\end{tabular}

Table:-5 Antibacterial Activity of Compounds (4a-e)

\begin{tabular}{|c|c|c|c|c|}
\hline \multirow{2}{*}{ Compounds } & \multicolumn{2}{|c|}{ Gram +Ve } & \multicolumn{2}{c|}{ Gram -Ve } \\
\cline { 2 - 5 } & $\begin{array}{c}\text { Staphylococcus } \\
\text { aureus }\end{array}$ & Bacillus subtilis & E.coli & Klebsiella promioe \\
\hline $4 \mathbf{4}$ & 56 & 55 & 59 & 52 \\
\hline $4 \mathbf{b}$ & 57 & 57 & 58 & 59 \\
\hline $\mathbf{4 c}$ & 59 & 58 & 68 & 56 \\
\hline $\mathbf{4 d}$ & 63 & 59 & 67 & 54 \\
\hline $4 \mathbf{4}$ & 70 & 70 & 79 & 67 \\
\hline
\end{tabular}

Table:-6 Antibacterial Activity of Compounds (5a-e)

\begin{tabular}{|c|c|c|c|c|}
\hline \multirow{2}{*}{ Compounds } & \multicolumn{2}{|c|}{ Gram +Ve } & \multicolumn{2}{c|}{ Gram -Ve } \\
\cline { 2 - 5 } & $\begin{array}{c}\text { Staphylococcus } \\
\text { aureus }\end{array}$ & Bacillus subtilis & E.coli & Klebsiella promioe \\
\hline $\mathbf{5 a}$ & 58 & 57 & 61 & 54 \\
\hline $\mathbf{5 b}$ & 59 & 59 & 59 & 61 \\
\hline $\mathbf{5 c}$ & 61 & 60 & 69 & 58 \\
\hline $\mathbf{5 d}$ & 64 & 62 & 69 & 55 \\
\hline $\mathbf{5 e}$ & 72 & 71 & 81 & 69 \\
\hline
\end{tabular}




\section{International Advanced Research Journal in Science, Engineering and Technology}

Vol. 6, Issue 1, January 2019

\section{Antifungal Activities}

The fungicidal activity of all the compounds was studied at $1000 \mathrm{ppm}$ concentration in vitro. Plant pathogenic organisms used were Nigrospora Sp, Aspergillus niger, Botrydepladia thiobromine, and Rhizopus nigricum, Fusarium oxyporium. Potato dextrose agar (PDA) medium. Such a PDA medium was used as a cultural food ${ }^{20}$

The fungicidal activity measured in $\%$ age growth of inhibition of all the compound (3a-e, 4a-e and 5a-e) is shown in Tables-7- 9 .

Table:-7 Antifungal Activity of Compounds (3a-e)

\begin{tabular}{|c|c|c|c|c|c|}
\hline \multicolumn{7}{|c|}{ Zone of Inhibition at 1000 ppm (\%) } \\
\hline Compounds & $\begin{array}{c}\text { Nigrospora } \\
\text { Sp. }\end{array}$ & $\begin{array}{c}\text { Aspergillus } \\
\text { Niger }\end{array}$ & $\begin{array}{c}\text { Botrydepladia } \\
\text { Thiobromine }\end{array}$ & $\begin{array}{c}\text { Rhizopus } \\
\text { Nigricum }\end{array}$ & $\begin{array}{c}\text { Fusarium } \\
\text { oxyporium }\end{array}$ \\
\hline 3a & 58 & 51 & 60 & 56 & 66 \\
\hline 3b & 67 & 68 & 61 & 61 & 67 \\
\hline 3c & 67 & 65 & 68 & 60 & 65 \\
\hline 3d & 66 & 66 & 69 & 71 & 63 \\
\hline 3e & 69 & 70 & 71 & 76 & 75 \\
\hline
\end{tabular}

Table:-8 Antifungal Activity of Compounds (4a-e)

\begin{tabular}{|c|c|c|c|c|c|}
\hline \multicolumn{7}{|c|}{ Zone of Inhibition at 1000 ppm (\%) } \\
\hline Compounds & $\begin{array}{c}\text { Nigrospora } \\
\text { Sp. }\end{array}$ & $\begin{array}{c}\text { Aspergillus } \\
\text { Niger }\end{array}$ & $\begin{array}{c}\text { Botrydepladia } \\
\text { Thiobromine }\end{array}$ & $\begin{array}{c}\text { Rhizopus } \\
\text { Nigricum }\end{array}$ & $\begin{array}{c}\text { Fusarium } \\
\text { oxyporium }\end{array}$ \\
\hline 4a & 60 & 53 & 62 & 58 & 67 \\
\hline 4b & 68 & 69 & 64 & 67 & 69 \\
\hline $4 \mathbf{4 c}$ & 71 & 68 & 72 & 63 & 68 \\
\hline 4d & 68 & 67 & 71 & 73 & 65 \\
\hline $4 \mathbf{4 e}$ & 72 & 73 & 74 & 78 & 77 \\
\hline
\end{tabular}

Table:-9 Antifungal Activity of Compounds (5a-e)

\begin{tabular}{|c|c|c|c|c|c|}
\hline \multicolumn{7}{|c|}{ Zone of Inhibition at 1000 ppm (\%) } \\
\hline Compounds & $\begin{array}{c}\text { Nigrospora } \\
\text { Sp. }\end{array}$ & $\begin{array}{c}\text { Aspergillus } \\
\text { Niger }\end{array}$ & $\begin{array}{c}\text { Botrydepladia } \\
\text { Thiobromine }\end{array}$ & $\begin{array}{c}\text { Rhizopus } \\
\text { Nigricum }\end{array}$ & $\begin{array}{c}\text { Fusarium } \\
\text { oxyporium }\end{array}$ \\
\hline 5a & 62 & 55 & 63 & 60 & 68 \\
\hline 5b & 69 & 71 & 65 & 68 & 71 \\
\hline 5c & 73 & 69 & 74 & 65 & 69 \\
\hline 5d & 69 & 69 & 72 & 74 & 67 \\
\hline 5e & 74 & 75 & 76 & 79 & 79 \\
\hline
\end{tabular}

\section{RESULTS AND DISCUSSION}

It was performed that Isoniazid (1), on condensation with 5-Arylfuran-2-carbaldehydes (2a-e), yields N'-((5-Arylfuran2-yl) methylene) isonicotinohydrazide (3a-e). There structures of (3a-e) were assigned by elemental analysis and IR and NMR spectra further the IR band at 1620-1640 (C=N), 3030-3080 $\mathrm{cm}^{-1}(\mathrm{C}-\mathrm{H}$, of Ar.), 1675-1685(C=O), 1185(C$\mathrm{O}-\mathrm{C}), 2950,1370 \mathrm{~cm}^{-1}\left(-\mathrm{CH}_{3}\right), 1085(-\mathrm{Cl}), 1550,1370\left(-\mathrm{NO}_{2}\right) .{ }^{1} \mathrm{H}$ NMR signals: $6.6-8.9(10 \mathrm{H}, \mathrm{m}, \mathrm{Ar}-\mathrm{H}), 11.8-11.9$ $(1 \mathrm{H}, \mathrm{s},-\mathrm{CONH}), 8.4-8.8(1 \mathrm{H}, \mathrm{s},-\mathrm{N}=\mathrm{CH}), 3 \mathrm{~b} ; 2.41\left(3 \mathrm{H}, \mathrm{s},-\mathrm{CH}_{3}\right)$. The $\mathrm{C}, \mathrm{H}, \mathrm{N}$ analysis data of all compounds are presented in Table -1 .

The structures assigned to N-(4-oxo-2-(5-Arylfuran-2-yl)thiazolidin-3-yl) isonicotinamide(4a-e) were supported by the elemental analysis and IR spectra showing an absorption bands at $1690 \mathrm{~cm}^{-1}(\mathrm{C}=\mathrm{O}$ of thiazolidinone ring $), 718 \mathrm{~cm}{ }^{-1}(\mathrm{C}-$ $\mathrm{S}-\mathrm{C}$ of thiazolidinone ring), $3075-3095 \mathrm{~cm}^{-1}\left(\mathrm{CH}_{2}\right.$ of thiazolidinone ring), 3030-3080 $\mathrm{cm}^{-1}$ (C-H, of Ar.), $1675-1685$ $\mathrm{cm}^{-1}(-\mathrm{CONH}), 1185(\mathrm{C}-\mathrm{O}-\mathrm{C}), 1085(-\mathrm{Cl}), 1550,1370\left(-\mathrm{NO}_{2}\right), 2950,1370 \mathrm{~cm}^{-1}\left(-\mathrm{CH}_{3}\right) .{ }^{1} \mathrm{H}$ NMR: $3.85-3.95\left(2 \mathrm{H}, \mathrm{s},-\mathrm{CH}_{2}\right.$ of the ring), 5.95-5.96 (1H, s,-CH), $6.6-8.9(10 \mathrm{H}, \mathrm{m}, \mathrm{Ar}-\mathrm{H}), 8.1-8.2(1 \mathrm{H}, \mathrm{s},-\mathrm{CONH}), 4 \mathrm{~b} ; 2.41\left(3 \mathrm{H}, \mathrm{s},-\mathrm{CH}_{3}\right) . \mathrm{The} \mathrm{C}, \mathrm{H}$, $\mathrm{N}, \mathrm{S}$ analysis data of all compounds are presented in Table-2.

The structures assigned to N-(4-oxo-2-(5-Arylfuran-2-yl)thiazolidin-3-yl) isonicotinamide(5a-e) were supported by the elemental analysis and IR spectra showing an absorption bands at $1690 \mathrm{~cm}^{-1}(\mathrm{C}=\mathrm{O}$ of thiazolidinone ring $), 718 \mathrm{~cm}{ }^{-1}(\mathrm{C}-$ $\mathrm{S}-\mathrm{C}$ of thiazolidinone ring), $3075-3095 \mathrm{~cm}^{-1}\left(\mathrm{CH}_{2}\right.$ of thiazolidinone ring), 3030-3080 $\mathrm{cm}^{-1}$ (C-H, of Ar.), 1675-1685 
Vol. 6, Issue 1, January 2019

$\mathrm{cm}^{-1}$ (-CONH), $1185(\mathrm{C}-\mathrm{O}-\mathrm{C}), 1085(-\mathrm{Cl}), 1550,1370\left(-\mathrm{NO}_{2}\right) 2850,2950,1370 \mathrm{~cm}^{-1}\left(-\mathrm{CH}_{3}\right), 3372 \mathrm{~cm}^{-1}(\mathrm{NH}), 1365,1185$ $\mathrm{cm}^{-1} \quad\left(\mathrm{SO}_{2}\right) . \quad{ }^{1} \mathrm{H} \quad \mathrm{NMR}: \quad 3.85-3.95 \quad\left(2 \mathrm{H}, \quad \mathrm{s},-\mathrm{CH}_{2} \quad\right.$ of the ring), 5.95-5.96 $\quad(1 \mathrm{H}, \quad \mathrm{s},-$ $\mathrm{CH}), 6.6-8.9(14 \mathrm{H}, \mathrm{m}, \mathrm{Ar}-\mathrm{H}), 8.1-8.2(1 \mathrm{H}, \mathrm{s},-\mathrm{CONH}), 4 \mathrm{~b} ; 2.41\left(3 \mathrm{H}, \mathrm{s},-\mathrm{CH}_{3}\right)$, The C, H, N, S analysis data of all compounds are presented in Table-2.

The examination of elemental analytical data reveals that the elemental contents are consistent with the predicted structure shown in Scheme-1. The IR data also direct for assignment of the predicted structure. The final structure of all compounds is confirmed by LC-MS. LC-MS data of all compounds are presented in Tables-1,2 and 3.

\section{CONCLUSION}

The reaction of Isoniazid (1) with 5-Arylfuran-2-carbaldehydes(2a-e), yields Schiff bases of N'-((5-Arylfuran-2yl)methylene)isonicotinohydrazide (3a-e), which on reaction with mercapto acetic acid yielded N-(4-oxo-2-(5Arylfuran-2-yl)thiazolidin-3-yl) isonicotinamide(4a-e) followed by hydrazo derivatives (5a-e), their structured were predicated by the elemental and spectral analysis. Newly prepared compounds were shows moderate to good antibacterial and antifungal activities.

\section{ACKNOWLEDGEMENT}

The authors are thankful to Department of Chemistry, Rabindranath Tagore University, Bhopal, (M.P.) for providing laboratory facilities.

\section{REFERENCES}

[1] Mostafa A., Nassar Y., Ibrahim S. Ahmed, Hasssan A. Dessouki, Sobhia S. AliJ. Bas. \& Environ. Sci., 5, 60. (2018)

[2] Kilic.T., Ugras. H.I., Basaran I. and Cakir. U, J. Heterocyclic Chem., 43, 1679. (2016)

[3] Ambhure.R.U., Mirgan.S. R. e, Thombal D.U., Nawale. R. B., Marathe. R. P., Pawar. R. P. Mod. Org. Chem. Res., 2,1. (2017)

[4] Venugopala .K.N., Rao. G.K. and Pai. Sanjay. P.N., Journal of Pharmacology and Toxicology.,2(5), 481. (2007)

[5] Hegab .K.H., Elzahany. E.A., Khalil .S.K.H. and Youssef.K.N.S, Australian J. of Basic and Applied Science. 2(2), 210. (2008).

[6] Wei. X.Q., Yi. YI, Xie. M.G. and LU. Z.YChinese Chemical Letters. 15, 525. (2014)

[7] Schlitzer. M., Debski. N. and Euler. HJ.Heterocycl.Chem., 33, 1143.(1996).

[8] Aslam. S., Asif.N. Khan .M. N., Khan. M.A., Munawar. M.A. and Nasrullah .M., Asian J. of Chemistry,25(14),7738. (2013)

[9] Rao. B.S., Holla. B.S., Shridhara. K. and Akberal.P.M. I, IL Farmaco, 55,338. (2000).

[10] Ilavský. D, Kada. R., Štetinová .J., Zalibera. L. and Padour .J. Collect.Czech. Chem. Commun., 59, 444. (2004).

[11] Srivastava. S., Jain.A., and Srivastava. S.,J. Indian Chem.Soc., 83, 1118 .(2006)..

[12] Shetgiri.N. P. and Chitre .A. D..Ind .J .Chem. 45(B), 1308. (2016)

[13] Jadav .R, Srivastava. S and Srivastava. S. D., Chemistry, An Indian Journal, 1, 95. (2013)

[14] Plasencia. C., Daym. R., Wang.Q, Pinski.J, Burke T. R Jr., Quinn. D. I. and Neamati. N. Mol. Cancer Ther. 4(7) 1105. (2015)

[15] Shah. P.,Heterocyclic Letters, 6 (1), 111 .(2016).

[16] Al- Mawsawi LQ, Dayam. R., Taheri. L., Witvrouw. M., Debyser. Z., Neamati.NBioorg. Med. Chem. Lett. 17(23) 6472. (2017).

[17] Rondestvedt. C. Org. Reactions, 11, 180. (1960).

[18] Metwally. M. A., Fekri.A., Nagy. N., Amer.F. A., Am. J. of Chemistry, 22(5), 277-281. (2012).

[19] Ravindra, K.C.; Vagdevi, H.M. and Vaidya,V.P., ARKIVOC, XI,1,(2008). 